\title{
Below-bandgap excitation of bulk semiconductors by twisted light
}

\author{
G. F. Quinteiro \\ CONICET and Departamento de Física "J. J. Giambiagi", Universidad de Buenos Aires \\ Ciudad Universitaria, Pabellón I, C1428EGA Ciudad de Buenos Aires, Argentina
}

(Dated: September 29, 2018)

\begin{abstract}
I theoretically investigate the response of bulk semiconductors to excitation by twisted light below the energy bandgap. To this end, I modify a well-known model of light-semiconductor interaction to account for the conservation of the light's momentum. I show that the excited states can be thought of as a superposition of slightly perturbed exciton states undergoing a complex center-ofmass motion. In addition, the absorption would occur at a slightly shifted energy (compared to plane waves) and would exhibit complex spatial patterns in the polarization and current.

PACS numbers:
\end{abstract}

FINAL VERSION PUBLISHED IN EUROPHYSICS LETTERS EPL 91, 27002 (2010)

In the realm of semiconductor optics, a generalized practice is to neglect the photon's momentum in direct absorption/emission processes. This assumption, known as "vertical transitions"(VT) [1], has been applied with such success that sometimes the need to include the momentum is understimated. Exceptions are found in early and recent works, reporting theoretical and experimental results strongly dependending on the conservation of the linear momentum of the photon. In the late ' 50 Hopfield elaborated the theory of exciton-polaritons [2], and later he pointed out, in a different context, the existence of new selection rules not accounted for by VT[3] . Afterwards the photon drag effect [4] was proposed, experimentally verified and brought to the point of technological applications. More recently, the photon momentum has been incorporated in the equations of motion describing the dynamics of electrons and holes under the action of inhomogeneous light fields to treat lasers $[5]$; meanwhile, research in exciton-polaritons continue evolving [6].

In the last few decades there has been an increase in the number of studies on spatially inhomogeneous light fields, most important to this article is the case of optical vortices [7], and subsequently that of twisted light(TL) - light carrying orbital angular momentum(OAM) [8]. These have motivated studies of inhomogeneous lightmatter interaction in several fields [9], and likely part of the work in semiconductors cited above. In particular, theoretical work by myself and collaborators show the need to include the OAM of TL in above-bandgap excitation of semiconductor structures. 10 12]

From the point of view of applications, the tendency to miniaturization requires the manipulation of states having few particles, and the consideration of small quantities. Thus, what has been judgicioulsly neglected in the past, may acquire relevance in present-days technology.

The present work is motivated by the combination of the current interest in optical vortices, the long-standing attention to the light-matter interaction problem, and fi- nally the intention to gain control on the quantum level in semiconductor structures. In the following I explain the modifications introduced in a standard model of lightsemiconductors interaction, to account for the linear momentum or OAM of the light. Using this extended model I work out the familiar situation of excitation of excitons by plane waves. I have in mind two aims; first, I intend to further support - in addition to the aforemention literature - the idea that the inclusion of the momentum of light is a must, for certain situations. Second, some simple new results and connection to other theories will emerge. The main part of the article treats the belowbandgap coherent excitation by TL.

The theory of optical excitation of semiconductors in the VT limit is well developed and known. An excellent treatment is given by Haug et al[1], for the cases without (inter-band transitions) and with (excitonic transitions) Coulomb interaction. In the following, this formalism will be extended to include the momentum of light.

The dynamics of electrons in a semiconductor having a valence $(v)$ and a conduction $(c)$ bands may be described by Heisenberg equations of motion [12] for populations in each band plus the quantum coherence between them; these equations form a coupled system. Under the condition of low excitation (low light field intensity/large detuning), the equations can be treated perturbatively and can be decoupled. [13] The first order term in the light field is the quantum interband coherence $\rho_{v i, c j}=\left\langle a_{v i}^{\dagger} a_{c j}\right\rangle$, where $a_{b k}^{\dagger} / a_{b k}$ are creation/annihilation operators of electrons in a Bloch state $\mathbf{k}$ and band $b$ : $\psi_{b \mathbf{k}}(\mathbf{r})=\langle\mathbf{r} \mid b \mathbf{k}\rangle=e^{i \mathbf{k r}} u_{b}(\mathbf{r})$, with energy $\varepsilon_{b \mathbf{k}}$, and periodic Bloch function $u_{b}(\mathbf{r})$; whereas, populations are second order in the light field. Then, the linear response of the unexcited system (zero conduction band population) is obtained from

$$
\begin{array}{r}
\left(i \hbar \frac{d}{d t}-\Delta_{c \mathbf{k}, v \mathbf{k}^{\prime}}\right) \rho_{v \mathbf{k}^{\prime}, c \mathbf{k}}(t)=\left\langle c \mathbf{k}\left|h_{I}(t)\right| v \mathbf{k}^{\prime}\right\rangle- \\
\sum_{\mathbf{q} \neq 0} V_{\mathbf{q}} \rho_{v \mathbf{k}^{\prime}-\mathbf{q}, c \mathbf{k}-\mathbf{q}}(t),
\end{array}
$$

where $\Delta_{c \mathbf{k}, v \mathbf{k}^{\prime}}=\varepsilon_{c \mathbf{k}}-\varepsilon_{v \mathbf{k}^{\prime}}$ and the self-energy correction 
has been neglected. The first term in the RHS is the matrix element of the light-matter interaction, modeled by "minimal coupling" (for the case of TL see Quinteiro et al [10]). The second term on the RHS is the Coulomb potential in the random phase approximation. I first note that, due to the conservation of the momentum of light, $\left\langle c \mathbf{k}\left|h_{I}(t)\right| v \mathbf{k}^{\prime}\right\rangle$ vanishes unless $\mathbf{k}^{\prime}=\mathbf{k}-\mathbf{q}_{0}$ for some vector $\mathbf{q}_{0}$. In the case of plane waves propagating along the $z$-axis $\mathbf{q}_{0}=q_{0} \hat{z}$ is a constant or parameter - the linear momentum of the photon-, while for twisted light $\mathbf{q}_{0}=q_{\|} \cos \theta \hat{x}+q_{\|} \sin \theta \hat{y}+q_{z} \hat{z}$ with $\theta$ a variable and $\left\{q_{\|}, q_{z}\right\}$ parameters (see below for details). Therefore, to capture the relevant physics of the light-matter interaction I specialize and study Eq. (1) at $\mathbf{k}^{\prime}=\mathbf{k}-\mathbf{q}_{0}$. I will treat the case of TL, since that of plane waves can be easily deduced from the former. The main contribution to the light-matter interaction arises from the transverse component $\mathbf{A}(\mathbf{r}, t)=\boldsymbol{\epsilon}_{\sigma} A_{0}(t) J_{l}\left(q_{\|} r_{\|}\right) \exp \left[i\left(q_{z} z+l \phi\right)\right]+$ c.c of the vector potential, with $J_{l}(x)$ a Bessel function, $\hbar l$ the OAM, $\boldsymbol{\epsilon}_{\sigma}$ the vector for circular polarization $\sigma=+/-$, and $q_{\|}<q_{z}$. A Fourier transform in k-space $\left\{f(\mathbf{r})=[L /(2 \pi)]^{3} \int d^{3} k e^{-i \mathbf{k r}} f(\mathbf{k})\right\}$ and time $\left[f(t)=1 /(2 \pi) \int d \omega e^{-i \omega t} f(\omega)\right]$ is applied to simplify each term: The light-matter interaction $\left\langle c \mathbf{k}\left|h_{I}(t)\right| v \mathbf{k}^{\prime}\right\rangle=$ $\xi(t) \delta_{\boldsymbol{\kappa}_{\|} q_{\|}} \delta_{\boldsymbol{\kappa}_{z} q_{z}} \exp (i \theta l) /\left(L q_{\|}\right)$becomes

$$
\left\langle c \mathbf{k}\left|h_{I}(t)\right| v \mathbf{k}-\mathbf{q}_{0}\right\rangle \rightarrow L^{3} \xi(\omega) \frac{e^{i \theta l}}{L q_{\|}} \delta(\mathbf{r})
$$

with $\xi(t)=-(-i)^{l}\left(\boldsymbol{\epsilon} \cdot \mathbf{p}_{c v}\right) Q A_{0}(t) / m, \mathbf{p}_{c v}=\left\langle u_{c}|\mathbf{p}| u_{c}\right\rangle$ the momentum-operator matrix element, $Q$ the electron's charge, $m$ the electron's mass, and $L$ the linear size of the system. Then,

$$
\begin{array}{r}
\Delta_{c \mathbf{k}, v \mathbf{k}-\mathbf{q}_{0}} \rho_{v \mathbf{k}-\mathbf{q}_{0}, c \mathbf{k}}(\omega) \rightarrow\left[\left(E_{g}+\frac{\hbar^{2} \mathbf{q}_{0}^{2}}{2\left|m_{v}^{*}\right|}\right)-\right. \\
\left.i \frac{\hbar^{2} \mathbf{q}_{0}}{\left|m_{v}^{*}\right|} \cdot \nabla-\frac{\hbar^{2}}{2 \mu} \nabla^{2}\right] \rho_{\mathbf{q}_{0}}(\omega, \mathbf{r})
\end{array}
$$

with $1 / \mu=1 /\left|m_{v}^{*}\right|+1 /\left|m_{c}^{*}\right|$, and the subscript $v / c$ from $\rho$ was eliminated to ease the notation. Finally,

$$
\frac{L^{3}}{(2 \pi)^{3}} \int d^{3} j V_{\mathbf{k}-\mathbf{j}} \rho_{v \mathbf{j}-\mathbf{q}_{0}, c \mathbf{j}}(\omega) \rightarrow V(\mathbf{r}) \rho_{\mathbf{q}_{0}}(\omega, \mathbf{r}) .
$$

Assembling all terms, the transformed version of Eq. (1) becomes

$$
\begin{gathered}
{\left[\hbar \omega-\left(E_{g}+\frac{\hbar^{2} \mathbf{q}_{0}^{2}}{2\left|m_{v}^{*}\right|}\right)+i \frac{\hbar^{2} \mathbf{q}_{0}}{\left|m_{v}^{*}\right|} \cdot \nabla+\frac{\hbar^{2}}{2 \mu} \nabla^{2}+\right.} \\
V(\mathbf{r})] \rho_{\mathbf{q}_{0}}(\omega, \mathbf{r})=L^{3} \xi(\omega) \frac{1}{L q_{\|}} e^{i \theta l} \delta(\mathbf{r})
\end{gathered}
$$

A comparison with the VT model $\left(\mathbf{q}_{0}=0\right)$ reveals two new terms: (1) $\hbar^{2} \mathbf{q}_{0}^{2} /\left(2\left|m_{v}^{*}\right|\right)$, and (2) $i \hbar^{2} /\left|m_{v}^{*}\right| \mathbf{q}_{0} \cdot \nabla$. Term (1) needs no special care, since it is a renormalization of the energy. Term (2) can be handled by either $i$ ) perturbation theory, or $i i$ ) completing squares and applying a unitary transformation (displacement in $\mathbf{k}$-space space) $U(\mathbf{r})=\exp \left[i\left(\mu /\left|m_{v}^{*}\right|\right) \mathbf{q}_{0} \cdot \mathbf{r}\right]$. I will mainly follow method $i i)$; however, the perturbation theory analysis yields useful information. First, I show that the perturbation is indeed small compared to the rest. The solutions to the unperturbed homogeneous equation [RHS of Eq. (4)] are the excitonic relative-motion wavefunctions $\psi_{\nu}(\mathbf{r})$ with energy $E_{\nu}$ and spatial extent $a_{B}^{*}$; then, $\hbar^{2} /\left|m_{v}^{*}\right| \mathbf{q}_{0} \cdot \nabla \psi_{\nu}(\mathbf{r}) \simeq \hbar^{2} q_{0} /\left(a_{B}^{*}\left|m_{v}^{*}\right|\right) \psi_{\nu}(\mathbf{r})$. Typically (e.g. GaAs) $a_{B}^{*} \simeq 10 \mathrm{~nm}$ and $q_{0} \simeq 10^{-2} \mathrm{~nm}^{-1}$, so $\hbar^{2} q_{0} /\left(a_{B}^{*}\left|m_{v}^{*}\right|\right) \simeq 0.1 \mathrm{meV}$ which is smaller than $E_{\nu} \simeq 1$ meV, as required. The ratio $(2) /(1) \simeq 8$, so I retain both terms. By method $i i)$ Eq. (4i) becomes

$$
\begin{gathered}
{\left[\hbar \omega-E_{g}-\frac{\hbar^{2} \mathbf{q}_{0}^{2}}{2 M}-\frac{\mathbf{p}^{2}}{2 \mu}+V(\mathbf{r})\right] \widetilde{\rho}_{\mathbf{q}_{0}}(\omega, \mathbf{r})=} \\
L^{3} \xi(\omega) \frac{e^{i \theta l}}{L q_{\|}} U(\mathbf{r}) \delta(\mathbf{r}),
\end{gathered}
$$

where $\widetilde{\rho}_{\mathbf{q}_{0}}(\omega, \mathbf{r})=U(\mathbf{r}) \rho_{\mathbf{q}_{0}}(\omega, \mathbf{r})$, and $M=\left|m_{v}^{*}\right|+\left|m_{c}^{*}\right|$. Redefining $E_{g}^{\prime}=E_{g}+\hbar^{2} \mathbf{q}_{0}^{2} /(2 M)$ the homogeneous part becomes the equation for the relative motion of an exciton. Back to $\mathbf{k}$-space, the complete solution is [14]

$$
\begin{aligned}
& \rho_{v \mathbf{k}-\mathbf{q}_{0}, c \mathbf{k}}(\omega)=L^{3} \xi(\omega) \frac{e^{i \theta l}}{L q_{\|}} \times \\
& \sum_{\nu} \frac{\psi_{\nu}^{*}(\mathbf{r}=0)}{\hbar \omega-E_{g}-\frac{\hbar^{2} \mathbf{q}_{0}^{2}}{2 M}-E_{\nu}} \psi_{\nu}\left(\mathbf{k}-\frac{\mu}{\left|m_{v}^{*}\right|} \mathbf{q}_{0}\right) .
\end{aligned}
$$

Later, it will prove useful a Taylor expansion of $\psi_{\nu}(\mathbf{k}-$ $\left.\mu \mathbf{q}_{0} /\left|m_{v}^{*}\right|\right)$, which is possible since each term is of the order of $\psi_{\nu}(\mathbf{k})\left(q_{0} a_{B}^{*} \mu /\left|m_{v}^{*}\right|\right)^{n}$, with $q_{0} a_{B}^{*} \mu /\left|m_{v}^{*}\right|<1$. Notice the correspondence between this expansion and the perturbation theory approach $i$ ).

Equation (6) is the building-block for several quantities describing both the electrons' kinetics/dynamics and the effect that electrons have on the EM-field. Next, I provide the expectation values of the polarization, the electric current, and the OAM. 15]

As a consequence of the conservation of photon momentum, the global polarization of the system is zero; thus, a more correct quantity is a local or spacedependent polarization

$$
\mathbf{P}(\mathbf{R}, t)=2\left(\frac{L_{\mathcal{R}}}{L}\right)^{3} \sum_{\mathbf{k} \mathbf{q}_{0}} \Re\left\{e^{i \mathbf{q}_{0} \cdot \mathbf{R}} \mathbf{d}_{v c} \rho_{v \mathbf{k}-\mathbf{q}_{0}, c \mathbf{k}}(t)\right\},(7)
$$

with $\Re\{\ldots\}$ the real part, $\mathbf{d}_{v c}$ the dipole matrix element, and $\mathbf{R}$ pointing to a macroscopic cell of linear size $L_{\mathbf{R}}$ small compared to the scale of variation of the EM-field, but larger than the unit cell of the semiconductor. In addition, a TL-field induces electric currents [10, 11]

$$
\mathbf{j}(\mathbf{R}, t)=2 \frac{Q}{m} \frac{1}{L^{3}} \sum_{\mathbf{k} \mathbf{q}_{0}} \Re\left\{e^{i \mathbf{q}_{0} \cdot \mathbf{R}} \mathbf{p}_{v c} \rho_{v \mathbf{k}-\mathbf{q}_{0}, c \mathbf{k}}(t)\right\} .
$$


The similarity between the polarization and current is not surprising; the theory of macroscopic media relates the polarization charge to the electric current by the continuity equation. Either one or the other may be used to study the effect that the interband coherence Eq. (6) has on the EM-field. It is also meaningful to calculate to OAM acquired by the electrons. For reasons similar to those that cause the global polarization to vanish, only an OAM in slices perpendicular to the propagation direction of light yields a non-zero result

$$
\begin{aligned}
L_{z}(Z, t)= & \beta e^{i q_{z} Z} \sum_{\mathbf{k} \mathbf{q}_{0}} \Re\left\{\left(p_{-, b^{\prime} b} e^{i \theta}+p_{+, b^{\prime} b} e^{-i \theta}\right) \times\right. \\
& \left.\rho_{v \mathbf{k}-\mathbf{q}_{0}, c \mathbf{k}}(t)\right\},
\end{aligned}
$$

where $\beta=-\left(4 \pi / q_{\|}\right) J_{2}\left(q_{\|} L\right)$, and $p_{ \pm, b^{\prime} b}=p_{x, b^{\prime} b} \pm p_{y, b^{\prime} b}$. Some general comments are in order: $i$ ) Because of their local character, all quantities exhibit spatial dependence; ii) to obtain explicit expressions, it only remains to insert the time-domain version of $\rho_{v \mathbf{k}-\mathbf{q}_{0}, c \mathbf{k}}(\omega)$ into Eqs. (17)(9), or to transform all quantities to the frequency domain and use $\rho_{v \mathbf{k}-\mathbf{q}_{0}, c \mathbf{k}}(\omega)$ directly; iii) for plane waves all sums over $\left\{\mathbf{k}, \mathbf{q}_{0}\right\}$ simplify to $\{\mathbf{k}\}$, while for TL they simplify to $\{\mathbf{k}, \theta\}$.

With the tools developed so far, I analyze the cases of plane waves and twisted light.

Plane waves: I set $l=0$ and $e^{i \theta l} /\left(L q_{\|}\right)=1$. The simplest situation is that of $q_{0}=0$, i.e. VT. By noting that $\sum_{k} \psi_{\nu}(\mathbf{k}) \propto \psi_{\nu}(\mathbf{r}=0)$ the expressions for the coherence and the polarization boil down to those of the standard result, that is the polarization is homogeneous and new absorption lines below the conductionband edge appear due to the exciton's binding energy $E_{\nu}$. Let us now turn to the general case $\mathbf{q}_{0}=q_{0} \hat{z}$, and examine Eq. (6) after expanding $\psi_{\nu}\left(\mathbf{k}-\mu \mathbf{q}_{0} /\left|m_{v}^{*}\right|\right)=$ $\psi_{\nu}(\mathbf{k})+\sum 1 / n !\left(\mu q_{0} /\left|m_{v}^{*}\right|\right)^{n} \partial_{k_{z}}^{n} \psi_{\nu}(\mathbf{k})$. The first term is similar to that of VTs; nevertheless, the presence of the kinetic energy term $\hbar^{2} q_{0}^{2} /(2 M)$ in the denominator signals the center-of-mass (COM) motion of the exciton, as has been known for long time. The next terms in the expansion of Eq. (6) are the consequences of the displacement in momentum space of the excitonic relative-motion wave-function, as seen by the argument of $\psi_{\nu}\left(\mathbf{k}-\mu \mathbf{q}_{0} /\left|m_{v}^{*}\right|\right)$. From the point of view of perturbation theory, the term $i \hbar^{2} /\left|m_{v}^{*}\right| \mathbf{q}_{0} \cdot \nabla$ in Eq. (4) causes the eigenstates to be a superposition of unperturbed excitonic wave-functions. Both standpoints tell us that this correction affects the internal degree of freedom, in contrast to the effect $\hbar^{2} \mathbf{q}_{0}^{2} /\left(2\left|m_{v}^{*}\right|\right)$ has. Shifting to the analysis of derived quantities, we see that the polarization presents spatial dependency on the coordinate $Z$; from this expression an electric susceptibility in the direction of the electric field can be deduced. Expanding the real part in Eq. (7) and after some calculation

$$
\begin{array}{r}
\chi(\omega)=-\left|d_{v c}\right|^{2} \sum_{\nu} \frac{\psi_{\nu}^{*}(\mathbf{r}=0)}{\hbar \omega-E_{g}-\frac{\hbar^{2} q_{0}^{2}}{2 M}-E_{\nu}}\left[\psi_{\nu}(\mathbf{r}=0)+\right. \\
\left.\sum_{n=1}^{\infty} \frac{1}{n !}\left(\frac{\mu q_{0}}{\left|m_{v}^{*}\right|}\right)^{n}\left(\frac{L}{2 \pi}\right)^{3} \int d^{3} \mathbf{k} \partial_{k_{z}}^{n} \psi_{\nu}(\mathbf{k})\right]+\ldots(10)
\end{array}
$$

where the dots indicate an extra term arising from $\left\{e^{i \mathbf{q} 0 \cdot \mathbf{R}} \mathbf{d}_{v c} \rho_{v \mathbf{k}-\mathbf{q}_{0}, c \mathbf{k}}(t)\right\}^{*}$. The susceptibility shows the expected features, i.e. no spatial dependence [16] but "spatial dispersion" ( $q_{0}$ dependence). The COM-motion correction in the denominator introduces a tiny shift in the absorption line. Used in conjunction with Maxwell's equations, the susceptibility yields information about the effect that electrons has on the EM field, e.g. attenuation of the beam. I finally note that the effects introduced by a finite wave-vector $q_{0}$ is negligible when the semiconductor's $z$-length is smaller than $2 \pi / q_{0}$, e.g. thin samples/quantum wells.

Twisted light: The full solution Eq. (6) having $e^{i \theta l} /\left(L q_{\|}\right) \neq 0$, and variable $\theta$ is used. Let us first focus on the term $\hbar^{2} q_{0}^{2} /(2 M)$. Regarded as the COM kinetic energy of excitons, it indicates a complex motion connected to both parameters $q_{z}$ and $q_{\|}$. Although $q_{\|}$is not a momentum (it relates to the inverse of the beam waist), its presence may be understood by thinking on the linear momentum $\left(\mathbf{P}^{(T L)}=\epsilon_{0} \mathbf{E} \times \mathbf{B}\right)$ at each point in space carried by the TL field: A preliminary calculation, under the assumption $q_{\|}<q_{z}$, shows that the radial and angular components of $\mathbf{P}^{(T L)}$ are proportional to $q_{\|}$, while - as expected - its $z$ component is proportional to $q_{z}$. Despite this tells that $q_{\|}$participates in the momentum, a deeper analysis remains to be done in order to confirm and expand this result. Once again, the argument of $\psi_{\nu}\left(\mathbf{k}-\mu \mathbf{q}_{0} /\left|m_{v}^{*}\right|\right)$ indicates that the TL-field excites states which are not exactly but slightly perturbed excitonic states. Additionally, the presence of the factor $e^{i \theta l}$ signals that a superposition of states - differing in their $\theta$ variable and thus having different phases - is created, in contrast to the case of the one-state excitation by plane waves. As for the macroscopic description of the system, the states contribute, by the term $E_{\nu}+\hbar^{2} q_{0}^{2} /(2 M)$, absorption lines below the band gap; the shift due to the COM kinetic energy is about $10 \mu \mathrm{eV}$. The absorption could be studied more carefully by either calculating the local polarization or the electric current, and if possible deriving a susceptibility or conductivity (through Ohm's law) respectively. It is of interest to look at the electric current produced by the interband coherence; using Eq. (6) and defining $\rho_{v \mathbf{k}-\mathbf{q}_{0}, c \mathbf{k}}(t)=e^{i \theta l} \rho_{0}(t)$ Eq. (8) becomes

$\mathbf{j}(Z, \Phi ; t)=\frac{Q}{m} \frac{4 \pi}{L^{3}} \sum_{\mathbf{k}} \Re\left\{i^{l} e^{i q_{z} Z} J_{l}\left(q_{\|} R\right) e^{i l \Phi} \mathbf{p}_{v c} \rho_{0}(t)\right\}$.

The current perpendicular to the $z$-axis exhibits complex flow patterns: for $l=1$ one observes circular flows 
around the beam axis, for $l=2$ two electric current vortices appear at both sides of the beam axis, for $l>2$ the complexity increases and several vortices show up (see Fig. 2 in Quinteiro et al [10]), suggesting a transfer of the optical vortices to the solid. As a consequence, the OAM in $z$-direction only exists for the case of $l= \pm 1$. To conclude, it is my believe that the the correct physical description of the TL-semiconductor interaction requires the inclusion of $\mathbf{q}_{0}$; this is simply because common semiconductor structures are not shaped to avoid the angular inhomogeneity of a TL beam. [17]

In conclusion, a modified version of a well-established model has been presented and used to explain the belowbandgap excitation of semiconductors by inhomogeneous light beams, with special emphasis on twisted light. First, I show how the standard results for the case of vertical transitions induced by plane waves are obtained in the appropriate limit; this attests for the robustness of the extended model. Next, the case of plane waves when the wave vector of the light beam is taken into account is analyzed; I show how to recover the exciton's COM motion, and an additional correction to the relative-motion wave-function is found. Then, the theory is applied to the case of twisted light. I predict that the optical excitation produces a superposition of states that differ slightly from excitons and undergo complex center-of-mass motion. In addition, I show that the absorption energy is modified by the center-of-mass motion, and that the polarization and electric current induced by the transition present a complex spatial pattern. Given the current interest in the interaction of inhomogeneous EM-fields with semiconductors, and in particular the work in excitonpolaritons, further research on the generation of excitonlike states and their complex motion due to twisted light excitation may significantly impact the basic research and applications in these areas.

I thank P. I. Tamborenea and J. Berakdar for fruitful discussions, and support by ANPCyT.

[1] H. Haug and S. W. Jauho, Quantum theory of the optical and electronic properties of semiconductors, Fourth Edition (World Scientific Publishing Company, Singapore, 2004).

[2] J. J. Hopfield, Phys. Rev. 112, 1555 (1958).

[3] J. J. Hopfield and D. G. Thomas, J. Phys. Chem. Solids 1960. Vol. 12 pp 276-284. D. G. Thomas, J. Phys. Chem. Solids 1960. Vol. 15 pp 86-96. Hopfield, J. J. and Thomas, D. G. Phys. Rev. Lett. 4, 357-359 (1960). Hopfield, J. J. and Thomas, D. G. Phys. Rev. 132, 563 (1963).

[4] A. F. Gibson and C. Walker, J. Phys. C:Solid State Phys., Vol 4, 1971. A F Gibson and S Montasser, J. Phys. C:Solid State Phys., Vol 8, 1975.

[5] Ortwin Hess and Tilmann Kuhn, Phys. Rev. A 54, 3347 (1996). Fausto Rossi and Tilmann Kuhn, Rev. Mod. Phys. 74, 895 (2002). M. Herbst, M. Glanemann, V. M. Axt, and T. Kuhn, Phys. Rev. B 67, 195305 (2003).

[6] G. Khitrova H. M. Gibbs, F. Jahnke, M. Kira, and S. W. Koch, Rev. of Mod. Phys., Vol. 71, No. 51591 (1999). G. F. Quinteiro, J. Fernández-Rossier, C. Piermarocchi, Phys. Rev. Lett. 97, 097401 (2006). G. F. Quinteiro, Phys. Rev. B 77, 075301 (2008). S. Portolan, O. Di Stefano, S. Savasta, and V. Savona, EPL 8820003 (2009). Iacopo Carusotto and Cristiano Ciuti, Phys. Rev. Lett. 93, 166401 (2004).

[7] David L. Andrews, Structured Light and Its Applications: An Introduction to Phase-Structured Beams and Nanoscale Optical Forces (Academic Press, 2008).

[8] L. Allen, M. W. Beijersbergen, R. J. C. Spreeuw, and J. P. Woerdman, Phys. Rev. A 45, 8185 (1992). L. Allen, M. J. Padgett, and M. Babiker, Prog. Opt. XXXIX, 291 (1999). M. Padgett, J. Courtial, and L. Allen, Phys. Today 57, Iss. 5, 35 (2004). G. Molina-Terriza, J. P. Torres, and L. Torner, Nature Phys. 3, 305 (2007).

[9] S. Al-Awfi and M. Babiker, Phys. Rev. A 61, 033401 (2000). B. L. C. Dávila-Romero, D. L. Andrews, and M. Babiker, J. Opt. B. Quantum Semiclass. Opt. 4, S66-S72 (2002). M. F. Andersen, C. Ryu, P. Clade, V. Natarajan, A. Vaziri, K. Helmerson, and W. D. Phillips, Phys. Rev. Lett. 97, 170406 (2006). T. P. Simula, N. Nygaard, S. X. Hu, L. A. Collins, B. I. Schneider, and K. Molmer, arXiv:0707.3698 1 [cond-mat.soft].

[10] G. F. Quinteiro and P. I. Tamborenea, EPL 85, 47001 (2009).

[11] G. F. Quinteiro and P. I. Tamborenea, Phys. Rev. B 79, 155450 (2009).

[12] G. F. Quinteiro and Jamal Berakdar, Opt. Express 17, 20465-20475 (2009).

[13] Perturbation theory breaks down when the quantization volumes for electrons and photons is the same and no additional dissipation channel exists. 2] On the other hand, the conservation of momentum requires that the system is larger than the wavelength $\lambda$ of light; thus, for a system smaller that the photon's quantization box and larger than $\lambda$ perturbation theory can be used.

[14] To account phenomenologically for decoherence $\omega$ may be replaced by $\omega+i \gamma$ in the denominator.

[15] These quantities were obtained from expressions in terms of field operators, and though straightforward, the derivation is lengthy and so not given.

[16] The $\exp \left(i q_{0} Z\right)$ was used to reconstruct the electric field in a formula of the type $P(Z)=\chi E(Z)$.

[17] An exception would be an arc-section sample, subtending a small angle, whose origin coincides with the the beam axis. 